\title{
Akutes Nierenversagen und Vaskulitis: Wann dran denken und was tun?
}

\author{
Benjamin Wilde, Anja Bienholz, Oliver Witzke, Andreas Kribben
}

Bei Patienten mit Autoimmunerkrankungen ist das Risiko für ein akutes Nierenversagen (ANV) besonders groß. So tritt bei bis zu $90 \%$ der Patienten mit ANCA-Vaskulitis ein intra-renales ANV auf. Eine schnelle und richtige Diagnose ist hier wichtig, um erhebliche Folgeschäden zu vermeiden. Doch wann sollte an eine Vaskulitis gedacht werden? Und wie sieht die richtige Therapie aus?

Hintergrund | Bei immunologischen Systemerkrankungen, einschließlich Vaskulitiden und systemischem Lupus erythematodes, kann ein akutes Nierenversagen (ANV) vom intrarenalen Typ auftreten [1]. Bei der ANCA-Vaskulitis (AAV) ist dies bei bis zu 90\% der Patienten der Fall [2-4]. Die Prognose des Patienten hängt im Wesentlichen ab von

- dem Ausmaß des ANV,

- der raschen Einleitung einer immunsuppressiven Therapie [4-9].

ANCA-Vaskulitis I Häufig verläuft das ANV klinisch zunächst relativ symptomarm [10]. Auch ein nephrotisches Syndrom tritt selten auf. Im Vordergrund stehen daher bei Erstkontakt mit dem Patienten die extrarenalen Organmanifestationen, die bei einer immunologischen Systemerkrankung häufig zu finden sind und richtig gedeutet werden müssen. Bei einer AAV sind dies beispielsweise konstitutionelle Symptome wie Fieber, Nachtschweiß und unklarer Gewichtsverlust $[11,12]$. Bestimmte Formen der AAV manifestieren sich vor allem im Kopfbereich. Die Patienten berichten über:

- sinusitische Beschwerden

- möglicherweise Hörverlust

- Uveitis/Episkleritis

- Epistaxis oder/und Hämoptysen [13, 14]

Lupus erythematodes | Beim systemischen Lupus erythematodes zeigen sich häufig eine B-Symptomatik sowie Hauterscheinungen(SchmetterlingsErythem) und/oder Ulzerationen [1].

IgA-Vaskulitis I Die IgA-Vaskulitis, vormals Purpura Schönlein-Henoch, zeichnet sich durch vaskulitische Hauterscheinungen in Form einer palpablen Purpura oder durch abdominelle Schmerzen aus [15].

Kryoglobulinämische Vaskulitis | Bei der kryoglobulinämischen Vaskulitis treten Hautnekrosen oder eine Minderperfusion von Endgliedern auf [15]. Sowohl bei ihr als auch bei der AAV kann zusätzlich eine palpable Purpura vorliegen.

\section{Akutes Nierenversagen: Wann an eine Vaskulitis denken?}

Definition ANV | Ein ANV liegt nach den KDIGOGuidelines vor, wenn

- das Serum-Kreatinin gegenüber dem Ausgangswert um mindestens $0,3 \mathrm{mg} / \mathrm{dl}$ innerhalb von 48 Stunden angestiegen ist oder

- sich das Serum-Kreatinin im Vergleich zu einem bekannten oder angenommenen Ausgangswert innerhalb von 7 Tagen um das 1,5-fache erhöht hat oder

- eine Oligurie bzw. Anurie vorliegt, die definiert ist als Urinausscheidung < 0,5 ml / $\mathrm{kg} \mathrm{KG} \mathrm{/} \mathrm{h} \mathrm{über}$ mindestens 6 Stunden $[16,17]$.

Einteilung I Das ANV kann nach der Ursache in drei Kategorien eingeteilt werden $(\triangleright$ Abb. 1) [16, 17]:

1. Prärenales ANV

2. (Intra-)Renales ANV

3. Postrenales ANV

Das postrenale ANV ist durch eine Abflussstörung des Harns bedingt. Zur Diagnose eines postrenalen ANV ist die Nieren-Sonographie Mittel der Wahl: Das Nierenbeckenkelchsystem zeigt im Falle eines postrenalen ANV einen Harnaufstau.

Prärenales ANV | Das prärenale ANV ist die Folge einer Minderperfusion der Niere, beispielsweise durch eine intravasale Volumendepletion. Bei der Untersuchung des Patienten sollte man daher den Volumenstatus überprüfen. Hilfreich sind Parameter wie:

- Blutdruck

- Puls und Hautturgor

- Status der Schleimhäute

Die Anamnese sollte mögliche Triggerfaktoren für Entgleisungen des Elektrolyt- und Wasserhaushaltes, wie Diarrhoen und Exsikkose, umfassen. Einen weiteren Anhaltspunkt bietet die Sonografie der Vena cava inferior, bei der der intravasale Volumenstatus ermittelt werden kann.

Intrarenales ANV | Falls weder ein prä- noch ein postrenales ANV vorliegt, sollte man an ein ANV 
vom intrarenalen Typ denken und diese Möglichkeit überprüfen $[16,17]$. Exogen toxische Ursachen für ein intrarenales ANV sind z. B. Kontrastmittel-Gabe und NSAR-Einnahme. Hier hilft die Anamnese des Patienten weiter:

- Lag kürzlich ein Krankenhausaufenthalt vor?

- Bestehen beispielsweise Gelenkschmerzen, die zur übermäßigen Schmerzmitteleinnahme geführt haben?

Differenzialdiagnostisch kommen auch endogentoxische Ursachen in Frage $(\bullet$ Abb. 1). Dazu zählen:

- Hyperurikämie

- Hämolyse

- Rhabdomyolyse

- Tumorlyse

- Hyperkalzämie

Aufschluss geben hier die korrespondierenden Laborwerte [16, 17].

\section{ANV bei immunologischen Systemerkrankungen und Vaskulitis}

Glomerulonephritis als Auslöser | Bei immunologischen Systemerkrankungen kommt es durch eine Glomerulonephritis (GN) zu einem intrarenalen ANV. Bei beinahe allen GN, die mit einer immunologischen Systemerkrankung zusammenhängen, ist ein nephritisches Sediment zu finden $[1,16,18]$.

Differenzialdiagnose | Differenzialdiagnostisch kommen in Frage:

- eine AAV

- ein anti-Glomerular-Basement-Membrane (anti-GBM)-Syndrom

- eine IgA-Vaskulitis

- eine Lupusnephritis

- eine kryoglobulinämische Vaskulitis [1,15-17]. Diese weisen zusätzliche klinische und serologische Merkmale auf ( $\bullet$ Tab. 1).

Urinanalyse I Bei Verdacht auf eine GN ist eine Urinanalyse notwendig. Typischerweise findet sich eine Mikrohämaturie und gegebenenfalls eine mäßige Proteinurie - selten im nephrotischen Ausmaß. Die phasenkontrastmikroskopische Untersuchung des Urinsediments zeigt bei einer GN Akanthozyten, d.h. stechapfelförmige Erythrozyten im nephritischen Sediment. Bei einer vaskulitis-assoziierten GN finden sich immer Akanthozyten.

Nierenbiopsie | Eine Nierenbiopsie sichert die Diagnose. Sie sollte auch dann durchgeführt werden, wenn weder die Urinanalyse noch die phasenkontrastmikroskopische Untersuchung zu einem Ergebnis geführt haben. Einige Formen der GN, wie z.B. die membranöse Glomerulonephritis, zeichnen sich durch ein nephrotisches Syndrom und nicht primär durch ein nephritisches Sediment aus $[15,18]$.

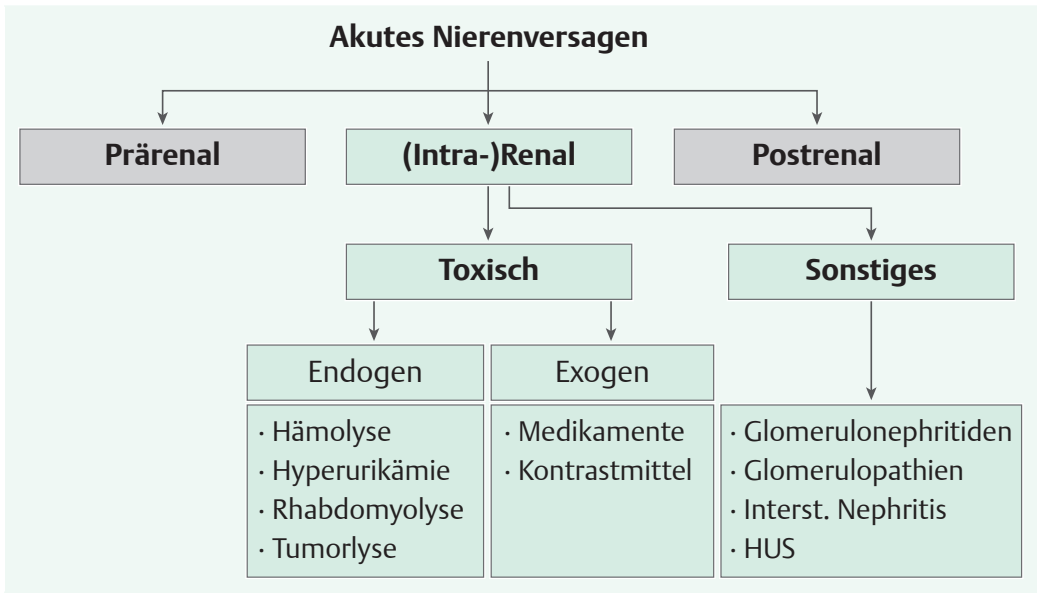

Abb. 1 Differenzialdiagnose des akuten Nierenversagens [33].

\section{Therapie der ANCA-assoziierten Vaskulitis}

Phasen der Therapie | Nach Empfehlung der European League Against Rheumatism (EULAR) gliedert sich die Therapie der AAV in zwei Phasen:

1. Induktionsphase

2. Erhaltungsphase $[19,20]$

In der Induktionsphase orientiert sich die Therapie an definierten Krankheitsstadien; in der Phase der Remissionserhaltung sollen Rezidive verhindert werden.

Einteilung in Kategorien | Die EULAR definiert 5 Krankheitskategorien bzw. -stadien, die die Krankheitsausprägung (abhängig von Art und Zahl der befallenen Organsysteme) beschreiben ( Tab. 2) [19, 20]:

1. lokalisiertes Stadium

2. frühsystemisches Stadium ohne organbedrohende Manifestation

3. generalisiertes Stadium mit organbedrohendem Befall

4. systemisches Stadium mit schwerem Organversagen

5. refraktäres Stadium

Bei einem ANV besteht ein Stadium 3 bis 5 .

Generalisiertes Stadium I Nach den EULARGuidelines liegt ein generalisiertes Stadium mit organbedrohender, renaler Manifestation erst ab einem Serum-Kreatinin-Wert von über $120 \mu \mathrm{mol} / 1$ (1,36 mg/dl) vor [11]. Der einzeitige Serum-Kreatinin-Wert alleine lässt jedoch keine Aussage über eine aktive Nierenbeteiligung oder ein ANV durch eine Glomerulonephritis zu. Um eine renale Krankheitsmanifestation zu beurteilen, sind das Vorhandensein eines nephritisches Sediments und der nierenbioptische Befund ausschlaggebend.

Die Kinetik des Serum-Kreatinins lässt auf ein ANV schließen. In den frühen Stadien der Nierenbeteiligung kommt es aber nicht immer zu einem ANV. In diesen Fällen ist das nephritische Sediment der 
Tab. 1 Akutes Nierenversagen bei immunologischen Systemerkrankungen und Vaskulitis: ausgewählte Differenzialdiagnosen. einzige Hinweis auf eine Glomerulonephritis. Die rapid-progressive nekrotisierende, pauci-immune Glomerulonephritis schreitet jedoch sehr rasch fort und führt zu einem schweren ANV.

Die Vaskulitis-assoziierte GN ist daher immer ein organbedrohender Befund und sollte mindestens dem Stadium „generalisiert" zugeordnet werden.

Induktionstherapie I In diesem Stadium muss eine Induktionstherapie mit Cyclophosphamid (CYC) oder Rituximab (RTX) erfolgen [21]. CYC wird in Form einer alters- und nierenfunktionsadpatierten Bolustherapie eingesetzt [21]. In der prospektiven, randomisierten CYCLOPS Studie wurde die zwei- bis drei-wöchentliche intravenöse CYC-Bolusgabe mit einem oralen CYC-Therapieschema zur Induktionstherapie verglichen [22]. Es konnte kein Unterschied hinsichtlich der Zeit bis zum Erreichen einer Remission festgestellt werden (im Median 3 Monate). Auch die Zahl der Rezidive im 18-monatigen Beobachtungszeitraum war - bei ähnlichem Nebenwirkungsprofil - statistisch nicht signifikant unterschiedlich (13 Rezidive Bolus-CYC, 6 Rezidive oral CYC) [22, 23].

Rituximab | Die Induktionstherapie mit Rituximab ist seit April 2013 in Deutschland zugelassen. Grundlage hierfür waren zwei randomisierte, placebo-kontrollierte Studien. Die Studie der EUVAS schließt GPA/MPA-Patienten mit schwerer Nierenbeteiligung (Mediane GFR $\sim 18 \mathrm{ml} / \mathrm{min}$ ) ein [24]. In dem einen $\operatorname{Arm}(n=11)$ erhielten die Patienten 6-8 CYC-Pulse ohne Rituximab; in einem anderen Arm $(n=33)$ erfolgte die Induktionstherapie mit zwei CYC-Stößen, gefolgt von vier Stößen Rituximab (RTX) [24]. 76\% der Patienten im RTX-Arm und $82 \%$ der Patienten im CYC-Arm erreichten nach 12 Monaten eine Remission $(P=0,67)$.

RAVE-Studie I Die sogenannte „RAVE“-Studie unterscheidet sich hinsichtlich des Studiendesigns. Außerdem wiesen die eingeschlossenen GPA/MPA-Patientenkohorten eine im Durchschnitt bessere Nierenfunktion (GFR um $60 \mathrm{ml} / \mathrm{min}$ ) auf [25]. Im CYC-Arm ( $=99)$ erhielten die Patienten orales CYC in Kombination mit Steroiden, im RTX-Arm wurden vier Pulse Rituximab verabreicht. 55\% der Patienten im CYCArm und 64\% der Patienten im RTX-Arm erreichten eine komplette Remission nach 6 Monaten $(p=0,21)[25]$. Insgesamt scheint die Induktionstherapie mit RTX einer Behandlung mit CYC nicht unterlegen zu sein.

Patienten im generalisierten Stadium mit Nierenbeteiligung sollten entweder mit CYC oder mit RTX - jeweils in Kombination mit Steroiden - behandelt werden.

Welche Therapieform man wählen sollte, ist abhängig von weiteren Faktoren, wie z.B. Lebensalter und Vorbehandlung.

Systemisches Stadium I Im schweren, systemischen Stadium der AAV zeigen sich vital bedrohende Manifestationen in der Regel mit einem ANV des Stadiums drei nach KDIGO. Das bedeutet, dass der Patient entweder

- aktuell dialysepflichtig ist und/oder

- der Serum-Kreatinin-Wert auf mindestens das 3-fache des Ausgangswerts innerhalb von 7 Tagen angestiegen ist [16, 17].

\begin{tabular}{|c|c|c|}
\hline & $\begin{array}{l}\text { Klinisches Erscheinungsbild } \\
\text { (ausgewählte Manifestationen) }\end{array}$ & Autoimmunserologie \\
\hline IgA-Vaskulitis & $\begin{array}{l}\text { Purpura, Arthralgien, Fieber } \\
\text { - Gastrointestinale Symptomatik (z. B. blutige } \\
\text { Durchfälle, Angina abdominalis) }\end{array}$ & \\
\hline Kryoglobulinämische Vaskulitis & $\begin{array}{l}\text { Assoziation mit Hepatitis B/C } \\
\text { - Assoziation mit anderen Autoimmunerkran- } \\
\text { kungen (z. B. Sjögren-Syndrom) oder } \\
\text { malignen Erkrankungen } \\
\text { - Purpura, Arthralgien, Polyneuropathien }\end{array}$ & - Kryoglobuline \\
\hline Lupus-Nephritis & $\begin{array}{l}\text { Hauterscheinungen (z. B. Schmetterlingsery- } \\
\text { them), Ulzerationen } \\
\text { - Arthralgien, B-Symptomatik }\end{array}$ & $\begin{array}{l}\text { - anti-dsDNA pos } \\
\text { - ANA-pos } \\
\text { C } 3, \text { C4 }\end{array}$ \\
\hline Anti-GBM-Krankheit & - Pulmorenales Syndrom & - anti-GBM pos \\
\hline ANCA-Vaskulitis & $\begin{array}{l}\text { Arthralgien / Arthritiden, B-Symptomatik, } \\
\text { evtl. Purpura } \\
\text { GPA: Episkleritis/Uveitis, Sinusitiden, } \\
\text { Epistaxis, Hämoptysen, Destruktionen des } \\
\text { Gesichtsschädels („Kopfklinik“) } \\
\text { MPA: evtl. Hämoptysen } \\
\text { - EGPA: Herzbeteiligung möglich }\end{array}$ & $\begin{array}{l}\text { ANCA-pos in den meisten Fällen mit } \\
\text { systemischer Erkrankung }\end{array}$ \\
\hline
\end{tabular}




\begin{tabular}{|c|c|c|c|c|}
\hline Krankheits-Kategorie & $\begin{array}{l}\text { Systemische Vaskulitis } \\
\text { (außerhalb oberer } \\
\text { Luftwege/Lunge) }\end{array}$ & $\begin{array}{l}\text { Organfunktion vital } \\
\text { bedroht }\end{array}$ & Anderes & $\begin{array}{l}\text { Nierenbeteiligung } \\
\text { (Serum-Kreatinin) }\end{array}$ \\
\hline Lokalisiert & Nein & Nein & $\begin{array}{l}\text { Keine konstitutionellen } \\
\text { (B-)Symptome } \\
\text { ANCA typischerweise } \\
\text { negativ }\end{array}$ & $\begin{array}{l}\text { Nein } \\
(<120 \mu \mathrm{mol} / \mathrm{l})\end{array}$ \\
\hline Früh systemisch & Ja & Nein & $\begin{array}{l}\text { Konstitutionelle (B-) } \\
\text { Symptome; ANCA positiv } \\
\text { oder negativ }\end{array}$ & $\begin{array}{l}\text { Möglich } \\
(<120 \mu \mathrm{mol} / \mathrm{l})\end{array}$ \\
\hline Generalisiert & $\mathrm{Ja}$ & Ja & ANCA positiv & $\begin{array}{l}\text { Ja } \\
(<500 \mu \mathrm{mol} / \mathrm{l})\end{array}$ \\
\hline Schwer systemisch & Ja & $\begin{array}{l}\text { Ja, schweres Organversa- } \\
\text { gen }\end{array}$ & ANCA positiv & $\begin{array}{l}\mathrm{Ja} \\
(>500 \mu \mathrm{mol} / \mathrm{l})\end{array}$ \\
\hline Refraktär & Ja & $\mathrm{Ja}$ & $\begin{array}{l}\text { Refraktär zur Standard- } \\
\text { therapie }\end{array}$ & - \\
\hline
\end{tabular}

Plasmapherese I Sinnvolle therapeutische Optionen sind extrakorporale Verfahren, wie z.B. der Plasmaaustausch. Pathogene Faktoren, wie Autoantikörper oder entzündungsfördernde Zytokine, werden so aus dem Blutkreislauf eliminiert. Der Einfluss der Plasmapherese auf Mortalität- und renales Überleben wurde in einer Kohorte von GPA/MPA Patienten mit schwerem ANV (Stadium $3, \mathrm{n}=137$ ) überprüft („MEPEX-Studie“) [26].

Die Patienten erhielten Plasmapherese zusätzlich zu einer CYC-basierten Induktionstherapie oder intravenöses Methylprednisolon zusätzlich zur CYC-basierten Induktionstherapie ohne Plasmapherese. In der Plasmapherese-Gruppe waren 69\% der Patienten nach 3 Monaten dialysefrei; im Kontrollarm ohne Plasmapherese waren es $49 \%$ der Patienten [26]. In der Nachbeobachtungsstudie war allerdings nach 4 Jahren keinen Unterschied zwischen den Gruppen hinsichtlich der Überlebensrate oder der Dialysefreiheit festzustellen [27].

Eine weitere randomisierte, kontrollierte Studie („PEXIVAS“) mit einer längeren Nachbeobachtungszeit und einem größeren Patientenkollektiv $(n=500)$ wird zurzeit durchgeführt [28].

Refraktäres Stadium | Zur Behandlung von Patienten mit refraktärem bzw. rezidivierendem Krankheitsverlauf liegen nur vereinzelt Studiendaten vor. Bei diesen Patienten ist unter bestimmten Bedingungen ein Wechsel der Präparate empfehlenswert. Das heißt nach primärer Induktionstherapie mit CYC, gefolgt von AZA-Erhaltungstherapie und anschließendem Rezidiv sollte ein RTX-basiertes Therapieregime erwogen werden [29].

In der RAVE-Studie zeigte sich, dass Patienten mit zuvor rezidivierendem Krankheitsverlauf unter RTX-Behandlung nach 6 und 12 Monaten eine geringere Rezdivrate aufwiesen als unter CYCTherapie ( 1 vs. 9 Rezidive; 0.004 vs. 0.034 pro „participant-month“, p=0.02; 0.014 vs. 0.033 Rezidive pro „participant-month“; $P=0.03)[25,29]$. Nach 18 Monaten hatte sich dieser Unterschied jedoch egalisiert (13 vs. 17 Rezidive; 0.018 vs. 0.027 pro „participant-month“, $\mathrm{P}=0.19)[25,29]$.

Erhaltungstherapie I Die AAV ist eine chronischrezidivierende Erkrankung, die zum teilweisen oder kompletten Verlust der Nierenfunktion führen kann [6, 30]. Eine Erhaltungstherapie muss sich der Induktionstherapie anschließen, um Rezidive zu verhindern und die Nierenfunktion zu erhalten. Die Erhaltungstherapie mit Azathioprin (AZA) nach Induktionstherapie mit CYC ist der fortgesetzten CYC-Therapie hinsichtlich Rezidivund Nebenwirkungsrate ebenbürtig und wird daher als Standard empfohlen [20,31].

\section{Akutes Nierenversagen bei Patienten mit bekannter Vaskulitis}

Besondere Überwachung I Der Patient mit bekannter AAV bedarf der besonderen ärztlichen Überwachung. Ein ANV sollte man wie beschrieben abklären. Gelenkschmerzen können ein NSAR-induziertes toxisches ANV begünstigen. Harnwegsinfekte können bei vorgeschädigten Nieren ebenfalls einen Anstieg der Retentionsparameter verursachen.

Rezidive der AAV sind häufig und können mit der Erstmanifestation einer Nierenbeteiligung einhergehen $[6,8,9]$.

Renale Rezidive I Isolierte renale Rezidive sind ebenfalls nicht selten. Renalen Rezidiven geht in vielen, aber nicht in allen Fällen, ein Anstieg des ANCA-Titers voraus [32]. Bei diesen Patienten ist Vorsicht geboten und der Urin sollte regelmäßig auf eine neu auftretende Mikrohämaturie bzw. ein nephritisches Sediment untersucht werden.
Tab. 2 Krankheitskategorien der ANCA-Vaskulitis nach den Empfehlungen der EULAR. 


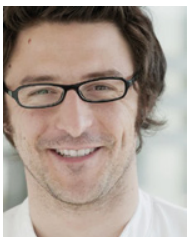

Priv.-Doz. Dr. med. Benjamin Wilde ist wissenschaftlicher Mitarbeiter an der Klinik für Nephrologie am Universitätsklinikum Essen.

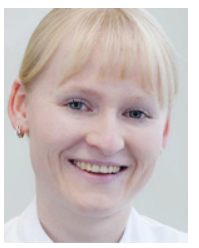

Dr. med. Anja Bienholz ist wissenschaftliche Mitarbeiterin an der Klinik für Nephrologie am Universitätsklinikum Essen.

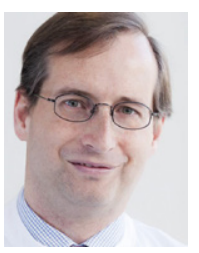

Prof. Dr. med. Oliver Witzke ist Vertreter des Direktors der Klinik für Nephrologie am Universitätsklinikum Essen.

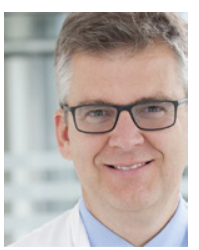

Prof. Dr. med. Andreas Kribben ist Direktor der Klinik für Nephrologie am Universitätsklinikum Essen. andreas.kribben@uni-due.de

DOI 10.1055/s-0041-100519 Dtsch Med Wochenschr 2015; 140: 256-260

(c) Georg Thieme Verlag KG . Stuttgart · New York .

ISSN 0012-0472
Ein alleiniger Anstieg der Autoantikörper rechtfertigt keine Therapieeskalation.

Die Mikrohämaturie kann dem Anstieg des Serum-Kreatinins als Zeichen des manifesten ANVs vorausgehen. Einige Patienten haben allerdings selbst in Phasen der klinischen Remission über Jahre eine persistierende Mikrohämaturie. Hier ist insbesondere auf eine Zunahme der Erythrozyturie bzw. der Akanthozyten im Urinsediment zu achten.

\section{Konsequenz für Klinik und Praxis}

- Bei Verdacht auf eine Nierenbeteiligung und/ oder bei akutem Nierenversagen ist eine Urinanalyse notwendig.

- Wenn eine Mikrohämaturie vorliegt, muss eine Glomerulonephritis erwogen werden.

- Eine Nierenbiopsie sichert die Diagnose. Extrarenale klinische Manifestationen können differentialdiagnostisch bedeutsam sein.

- Bei immunologischen Systemerkrankungen ist eine rasche Einleitung der immunsuppressiven Therapie notwendig.

- Patienten mit bekannter renaler Manifestation im Rahmen einer immunologischen Systemerkrankung müssen engmaschig überwacht werden.

\section{Literatur}

1 Couser WG. Basic and translational concepts of immune-mediated glomerular diseases. J Am Soc Nephrol 2012; 23: 381-399

2 Comarmond C, Cacoub P. Granulomatosis with polyangiitis (Wegener): Clinical aspects and treatment. Autoimmun Rev 2014; 13: 1121-1125

3 Kallenberg CGM. The diagnosis and classification of microscopic polyangiitis. J Autoimmun 2014; 48-49: 90-93

4 Sinico RA, Di Toma L, Radice A. Renal involvement in anti-neutrophil cytoplasmic autoantibody associated vasculitis. Autoimmun rev 2013; 12: 477-482.

5 Leib ES, Restivo C, Paulus HE. Immunosuppressive and corticosteroid therapy of polyarteritis nodosa. Am J Med 1979; 67: 941-947

6 Slot MC, Tervaert JW, Franssen CF, Stegeman CA. Rena survival and prognostic factors in patients with PR3-ANCA associated vasculitis with renal involvement. Kidney Int 2003; 63: 670-677

7 Wilde B, Van Paassen P, Witzke O, Cohen Tervaert JW. New pathophysiological insights and treatment of ANCA-associated vasculitis. Kidney Int 2011; 79: 599-612

8 Hilhorst M, Wilde B, van Breda Vriesman P et al. Estimating renal survival using the ANCA-associated GN classification. J Am Soc Nephrol 2013; 24: 1371-1375

9 Hilhorst M, Wilde B, van Paassen $\mathrm{P}$ et al. Improved outcome in anti-neutrophil cytoplasmic antibody (ANCA)-associated glomerulonephritis: a 30-year follow-up study. Nephrol Dial Transplant 2013; 28: 373-379

10 Flossmann O, Berden A, de Groot K et al. Long-term patient survival in ANCA-associated vasculitis. Ann Rheum Dis 2011; 70: 488-494

11 Hellmich B, Flossmann O, Gross WL et al. EULAR recommendations for conducting clinical studies and/or clinical trials in systemic vasculitis: focus on anti-neutrophil cytoplasm antibody-associated vasculitis. Ann Rheum Dis 2007; 66: 605-617
12 Mukhtyar C, Flossmann O, Hellmich B et al. Outcomes from studies of antineutrophil cytoplasm antibody associated vasculitis: a systematic review by the European League Against Rheumatism systemic vasculitis task force. Ann Rheum Dis 2008; 67: 1004-1010

13 Holle JU, Laudien M, Gross WL. Clinical manifestations and treatment of Wegener's granulomatosis. Rheum Dis Clin North Am 2010; 36: 507-526

14 Berden A, Goceroglu A, Jayne D et al. Diagnosis and management of ANCA associated vasculitis. Bmj 2012; 344: e26

15 Tarzi R, Harper L. Small vessel vasculitides. Medicine 2014; 42: 138-145

16 Bienholz A, Feldkamp T, Kribben A. Akutes Nierenversagen. Update 2013. Dtsch med Wochenschr 2013; 138: 1229-1232

17 Khwaja A. KDIGO Clinical Practice Guidelines for Acute Kidney Injury. Nephron Clin Pract 2012; 120: c179-c184

18 Segelmark M, Hellmark T. Autoimmune kidney diseases. Autoimmu rev 2010; 9: A366-A371

19 Hellmich B, Flossmann O, Gross WL et al. EULAR recommendations for conducting clinical studies and/ or clinical trials in systemic vasculitis: focus on anti-neutrophil cytoplasm antibody-associated vasculitis. Ann Rheum Dis 2007; 66: 605-617

20 Mukhtyar C, Guillevin L, Cid MC et al. EULAR recommendations for the management of primary small and medium vessel vasculitis. Ann Rheum Dis 2009; 68: 310-317

21 Ntatsaki E, Carruthers D, Chakravarty K et al. BSR and BHPR guideline for the management of adults with ANCA-associated vasculitis. Rheumatology 2014; 53 : 2306-2309

22 de Groot K, Harper L, Jayne DRW et al. Pulse Versus Daily Oral Cyclophosphamide for Induction of Remission in Antineutrophil Cytoplasmic Antibody Associated Vasculitis. Ann Intern Med 2009; 150: 670-680

23 Walsh M, Faurschou M, Berden A, F et al. Long-Term Follow-Up of Cyclophosphamide Compared with Azathioprine for Initial Maintenance Therapy in ANCA-Associated Vasculitis. Clin J Am Soc Nephrol 2014; 9: 1571-1576

24 Jones RB, Cohen Tervaert JW, Hauser T et al. Rituximab versus Cyclophosphamide in ANCA-Associated Renal Vasculitis. N Engl ] Med 2010; 363: 211-220

25 Stone JH, Merkel PA, Spiera R et al. Rituximab versus Cyclophosphamide for ANCA-Associated Vasculitis. N Engl J Med 2010; 363: 221-232

26 Jayne DRW, Gaskin G, Rasmussen N et al. Randomized Trial of Plasma Exchange or High-Dosage Methylprednisolone as Adjunctive Therapy for Severe Renal Vasculitis. J Am Soc Nephrol 2007; 18: 2180-2188

27 Walsh M, Casian A, Flossmann O et al. Long-term follow-up of patients with severe ANCA-associated vasculitis comparing plasma exchange to intravenous methylprednisolone treatment is unclear. Kidney Int 2013; 84: 397-402

\section{Interessenkonflikt}

B.W. gibt an, Reisespesen von Alexion, Chisei, TEVA und Wyeth erhalten zu haben.

A.B. gibt an Reisespesen von Alexion, Astellas und CLS Behring erhalten zu haben.

O.W. gibt an, Forschungsstipendien für klinische Studien, Redehonorare, Honorare und Reisespesen von Alexion, Amgen, Astellas, Bristol-Myers Squibb, Chiesi, Novartis, Roche, Pfizer und Sanofi erhalten zu haben.

A. K gibt an, Gelder für klinische Studien, Seminare, Redehonorare und Reisespesen von Alexion, Amgen, Astellas, Chiesi, Novartis, Roche, Fresenius und Sanofi erhalten zu haben. 
28 Walsh M, Merkel PA, Peh CA et al. Plasma exchange and glucocorticoid dosing in the treatment of anti-neutrophil cytoplasm antibody associated vasculitis (PEXIVAS): protocol for a randomized controlled trial. Trials 2013; 14: 73

29 Miloslavsky EM, Specks U, Merkel PA et al. Clinical outcomes of remission induction therapy for severe antineutrophil cytoplasmic antibody-associated vasculitis. Arthritis Rheum 2013; 65: 2441-2449

30 Slot MC, Tervaert JWC, Boomsma MM, Stegeman CA. Positive classic antineutrophil cytoplasmic antibody (C-ANCA) titer at switch to azathioprine therapy associated with relapse in proteinase 3-related vasculitis. Arthritis Rheum 2004; 51: 269-273.

31 Jayne D, Rasmussen N, Andrassy K et al. A Randomized Trial of Maintenance Therapy for Vasculitis Associated with Antineutrophil Cytoplasmic Autoantibodies. N Engl J Med 2003; 349: 36-44.

32 Kemna MJ, Damoiseaux J, Austen J et al. ANCA as a Predictor of Relapse: Useful in Patients with Renal Involvement But Not in Patients with Nonrenal Disease. J Am Soc Nephrol 2014.pii: ASN.2013111233

33 Feldkamp T, Gäckler A, Herget-Rosenthal S, Kribben A. State of the Art - Akutes Nierenversagen. Kompendium Nephrologie 2010, 2: 6-16 